\title{
Water insoluble polymers as efficient binder in fluid bed granulation of metoprolol for preparation hydrophilic matrix extended- release tablets
}

\author{
Dana HALES, Cristian ALECU, Ioan TOMUTA \\ Department of Pharmaceutical Technology and Biopharmacy, Faculty of Pharmacy, \\ "Iuliu Hatieganu" University of Medicine and Pharmacy, Cluj-Napoca, Romania
}

\begin{abstract}
Objectives. The objective of this study was to investigate the possibility of developing metoprolol extended-release tablets by using hydroxypropyl methylcellulose (HPMC) in order to obtain the hydrophilic matrix and Eudragit NE $40 D$, Kollicoat SR 30D and Surelease E7 as binders during the granulation process.

Material and methods. The extended-release tablets were prepared via fluid bed granulation of metoprolol powder using Eudragit NE 40D / Kollicoat SR 30D / Surelease E7 as binders, followed by compression. The influence of three formulation factors (the type of granulation polymers, the ratio of granulation polymers and the HPMC ratio) on the kinetic metoprolol tartrate release was investigated through a full factorial experimental design.

Outcomes. The kinetic release of all 26 formulations was best fitted with Peppas model. According to $n$ values of Peppas equation, the release mechanism of drug consists in water diffusion into the matrix, followed by matrix swelling and erosion. The results also indicated that the formulations containing an increased amount of Eudragit NE (10\% or more) as binder in the granulation process presented a satisfactory release rate of metoprolol over 12 hours from the granules incorporated in the hydrophilic matrix.

Conclusions. This study demonstrated the possibility of lowering of the burst effect from hydrophilic matrix extendedrelease dosage forms incorporating a freely soluble drug, by granulating the drug with a high amount of Eudragit NE $40 \mathrm{D}$ and processing the obtained granules in a hydrophilic matrix by tableting.
\end{abstract}

Keywords: metoprolol, hydrophilic matrix, extended-release, Eudragit NE, Kollicoat, Surelease, HPMC

\section{INTRODUCTION}

The extended-release oral drug delivery systems are characterized by their ability to drug release continuously, over an extended period of $12-24 \mathrm{~h}$, offering several advantages, such as improved dosage regimens, increased compliance, a better therapeutic effect and lower side-effects [1,2]. The most common type of extended-release oral dosage forms is the hydrophilic matrix, which is usually obtained from cellulose ethers such as hydroxypropyl cellulose (HPC), carboxymethyl cellulose (CMC), hydroxypropyl methylcellulose (HPMC) [3,4]. Hydroxypropyl 
methylcellulose (HPMC) is a semisynthetic polymer available in several grades that vary according to degree and ratio of substitution of the hydroxypropyl and methoxyl groups and therefore in hydration rate and water viscosity; it is widely used in pharmaceutical as matrix-forming polymer in preparation of extendedrelease oral tablets [4]. Even when high-viscosity grades of HPMC are used, obtaining hydrophilic matrix extended-release oral dosage forms with metoprolol (a freely wate- soluble drugs) is difficult due to initial high amount release [5]. The aqueous dispersions of water insoluble coating polymers as ethylcellulose (e.g. Aquacoat ECD, Surelease), methacrylic derivatives (e.g. Eudragit NE 40D) or polyvinyl acetate (e.g. Kollicoat SR 30D), create an insoluble but permeable film and are commonly used to coat pellets, granules, or tablets in order to obtain reservoir-type modified release oral dosage form [6-9].

Generally an initial burst effect can be observed when incorporating freely water-soluble drugs into the hydrophilic matrices. Therefore, adding waterinsoluble coating polymers as binders during the fluid bed granulation process of freely water-soluble drug in association with high-viscosity HPMC as matrixforming polymer could solve this problem by partially coating the freely water-soluble drug and thus reducing the penetration of water in the matrix, slowing the drug solubilization which will consequently determine a decreased of the initial drug diffusion with the reduction of the burst effect. The water-insoluble coating polymers that could be used as binders in fluid bed granulation of freely water-soluble drugs include, among others, polyvinyl acetate (Kollicoat SR) ethylcellulose (Surelease), and methacrylic acid copolymers (Eudragit NE) [10,11].

Design of experiments (DoE) is a powerful tool which reveals, through systematic approach, the most important formulation factors or process parameters that may affect the product characteristics. By using DoE, it is able to find, in a time and resource efficient manner, the level at which a factor determines the achievement of an optimum product and process [12-14].

The aim of this work was to investigate whether, it is possible, by using aqueous dispersions of insoluble coating polymers, Kollicoat, Eudragit and Surelease, as binders in preparation of granules via fluid bed granulation together with high viscosity HPMC (Methocel K100 M) as matrix-forming polymer in tableting, could reduce the burst effect of metoprolol, a freely water-soluble drug, in hydrophilic matrix extended-release tablets as oral dosage forms.

\section{MATERIAL AND METHODS}

\section{Materials}

Metoprolol tartrate (from Microsin, Romania) as drug substance; lactose monohydrate 200 mesh (from Meggle, Germany) and microcrystalline cellulose PH102 (from JRS, Germany) as filler in granulation;; hydroxypropyl methylcellulose (HPMC) - Methocel K100 M (from Colorcon, UK) as matrix forming; direct compressible lactose - Tablettose 80M (from Meggle, Germany) as filler in tableting; fumed silica - Aerosil 200 (from Degussa, Germany) and magnesium stearate (from Merck, Germany) as lowing agents; Kollicoat SR 30D (from BASF, Germany); Eudragit NE 40D (from Degussa, Germany); Surelease E7 19010 (from Colorcon, UK) insoluble coating polymers used as binding agents in fluid bed granulation.

\section{Design of experiment (DoE)}

A full factorial design of experiment (DoE) with three factors and three levels was used to study the influence of three formulation factors on the pharmaceutical characteristics of the hydrophilic matrix extended-release tablets. Table 1 illustrates the formulation factors and their level of variation, while table 2 shows the design of experiment matrix. Modde software (Sartorius Stedim Data Analytics AB, Sweden) was used to build the experimental design of the study, as well as to calculate the coefficients, statistical parameters and fitting of the experimental data [15].

\section{Granules preparation}

The granulation process was conducted in a Strea 1 fluid bed granulator (from Aeromatic A.G., Switzerland). The granulation formula and the processing conditions are presented in table 3 . After the binder solution spraying completed, the granules were maintained for another 30 minutes in the fluid bed granulator at $60^{\circ} \mathrm{C}$ and low fluidization air fan, in order to be properly dry the granules. According with design of experiment matrix, the ratios of the three insoluble polymers used as binders in aqueous dispersions (Kollicoat SR 30D, Eudragit NE 40D and Surelease E7) were $4 \%, 8 \%$ and $12 \%$.

\section{Tablets preparation}

The tablets (weight $450 \mathrm{mg}, 100 \mathrm{mg}$ metoprolol tartrate/tablet) were prepared using an eccentric 
TABLE 1. Formulation factors (independent variables) and their level of variation

\begin{tabular}{|l|c|c|c|c|}
\hline \multicolumn{1}{|c|}{ Variables } & Symbol & \multicolumn{3}{c|}{ Levels } \\
\cline { 3 - 5 } & $\mathrm{X}_{1}$ & -1 & 0 & +1 \\
\hline $\begin{array}{l}\text { Type of } \\
\text { granulation } \\
\text { polymer }\end{array}$ & $\mathrm{X}_{2}$ & 4 & 8 & 12 \\
\hline $\begin{array}{l}\text { Ratio of } \\
\text { granulation } \\
\text { polymer (\%) }\end{array}$ & & & & Kollicoat \\
\hline HPMC ratio (\%) & $\mathrm{X}_{3}$ & 20 & 30 & 40 \\
\hline
\end{tabular}

TABLE 3. Granulation formula and fluid bed granulator processing parameters

\begin{tabular}{|c|c|}
\hline Granulation formula & $\%(\mathrm{~m} / \mathrm{m}$ \\
\hline Metoprolol tartrate & $27.78 \%$ \\
\hline Lactose monohydrate & $30 \%$ \\
\hline $\begin{array}{l}\text { Binder (Eudragit NE 40D / Kollicoat SR } \\
\text { 30D / Surelease E7 19010* }\end{array}$ & $4-8-12 \%$ \\
\hline Purified water $* *$ & q.s. \\
\hline \multicolumn{2}{|l|}{$\begin{array}{l}\text { Fluid bed granulator processing } \\
\text { parameters }\end{array}$} \\
\hline $\begin{array}{l}\text { Binder solution spray rate - peristaltic } \\
\text { pump speed (rpm) }\end{array}$ & 10 \\
\hline Diameter of the gun nozzle $(\mathrm{mm})$ & 0.8 \\
\hline Atomization air pressure (atm.) & 1 \\
\hline Fan air (m3/min) & $3-5$ \\
\hline Inlet Air Temperature (OC) & 70 \\
\hline Outlet Air Temperature (OC) & $27-33$ \\
\hline Granulation process duration (min) & 25 \\
\hline
\end{tabular}

* according to the design of experiment matrix, see Table II

** needed to prepare a binder solution with $26 \%$ polymer content

tablet press EKO (from Korsch, Germany), equipped with $10 \mathrm{~mm}$ diameter lenticular set of punch and die. The composition of the extended-release tablets included: $50 \%$ metoprolol granules, $1 \%$ silicon dioxide, $1 \%$ magnesium stearate, $20-30-40 \%$ HPMC according with the design of experiment matrix, and the difference to $100 \%$ being represented by direct compressible lactose.

\section{Determination of the DoE's dependent variables}

The responses of the design of experiment (dependent variables) were the Peppas kinetic equation coefficients, $k$ and $n$ and the percents (\%) of metoprolol released at different time intervals $(1,2,3$, $4,6,8,12$ hours) during a period of 12 hours. The release of metoprolol from the extended-release tablets was evaluated according to the officinal procedure for European Pharmacopoeia ("2.9.3. Dissolution test for solid dosage forms"): The dissolution test was performed using the following
TABLE 2. Design of experiment (DoE) matrix

\begin{tabular}{|l|c|c|c|c|}
\hline $\begin{array}{c}\text { Experiment } \\
\text { Name }\end{array}$ & $\begin{array}{c}\text { Run } \\
\text { Order }\end{array}$ & X1 & X2 & X3 \\
\hline Exp 1 & 2 & Eudragit & 4 & 20 \\
\hline Exp 2 & 18 & Eudragit & 4 & 30 \\
\hline Exp 3 & 26 & Eudragit & 4 & 40 \\
\hline Exp 4 & 14 & Eudragit & 8 & 20 \\
\hline Exp 5 & 9 & Eudragit & 8 & 30 \\
\hline Exp 6 & 20 & Eudragit & 8 & 40 \\
\hline Exp 7 & 7 & Eudragit & 12 & 20 \\
\hline Exp 8 & 29 & Eudragit & 12 & 30 \\
\hline Exp 9 & 13 & Eudragit & 12 & 40 \\
\hline Exp 10 & 21 & Eudragit & 8 & 30 \\
\hline Exp 11 & 17 & Kollicoat & 4 & 20 \\
\hline Exp 12 & 11 & Kollicoat & 4 & 30 \\
\hline Exp 13 & 6 & Kollicoat & 4 & 40 \\
\hline Exp 14 & 25 & Kollicoat & 8 & 20 \\
\hline Exp 15 & 23 & Kollicoat & 8 & 30 \\
\hline Exp 16 & 4 & Kollicoat & 8 & 40 \\
\hline Exp 17 & 22 & Surelease & 4 & 20 \\
\hline Exp 18 & 12 & Surelease & 4 & 30 \\
\hline Exp 19 & 19 & Surelease & 4 & 40 \\
\hline Exp 20 & 3 & Surelease & 8 & 20 \\
\hline Exp 21 & 5 & Surelease & 8 & 30 \\
\hline Exp 22 & 16 & Surelease & 8 & 40 \\
\hline Exp 23 & Surelease & 12 & 20 \\
\hline Exp 24 & Surelease & 12 & 30 \\
\hline Exp 25 & Surelease & 12 & 40 \\
\hline Exp 26 & Surelease & 8 & 30 \\
\hline 16 15 & & \\
\hline
\end{tabular}

$\mathrm{X} 1$ - granulation polymer type, X2 - Ratio of granulation polymer, X3 - HPMC ratio

dissolution conditions: dissolution tester, apparatus no. 2 (paddle) from PharmaTest, Germany; dissolution medium: $900 \mathrm{~mL}$ phosphate buffer at $\mathrm{pH} 6.8$; dissolution temperature: $37^{\circ} \mathrm{C}$; agitation: $50 \mathrm{rpm}$ of the paddle; drug assay, using a validated UV spectrometric method at $275 \mathrm{~nm}$.

\section{Investigation of the release kinetics of the drug}

In order to determine the release kinetics of metoprolol from the extended-release tablets, the experimental data were fitted with the following mathematical equations: Korsmeyer-Peppas, Higuchi, zero order, Hixon-Crowell, Baker-Lonsdale, and first order (the equation are presented in table 4). In order to avoid overfitting only a value greater than $80 \%$ was considered when calculating the release kinetics [16-18]. Also, the correlation coefficient must be nearly $1[16,17]$ and the Akaike index must have the lowest value to consider that the release kinetics is adequate [18]. 
TABLE 4. Mathematical models of drug release

\begin{tabular}{|l|c|}
\hline Higuchi & $\mathrm{Qt} / \mathrm{Q} \infty=\mathrm{Kt} 0.5$ \\
\hline $\begin{array}{l}\text { Korsmeyer - } \\
\text { Peppas }\end{array}$ & $\mathrm{Qt} / \mathrm{Q} \infty=\mathrm{Ktn}$ \\
\hline Zero Order & $\mathrm{Qt}=\mathrm{Q} 0+\mathrm{Kt}$ \\
\hline Hixson-Crowell & $\mathrm{Q} 01 / 3-\mathrm{Qt} 1 / 3=\mathrm{Kt}$ \\
\hline Baker-Lonsdale & $\begin{array}{c}{[3 / 2][1-[1-[\mathrm{Qt} /} \\
\mathrm{Q} \infty] 2 / 3]-[\mathrm{Qt} / \mathrm{Q} \infty]=\mathrm{Kt}\end{array}$ \\
\hline First Order & $\mathrm{Qt} / \mathrm{Q} \infty=\mathrm{Kt}$ \\
\hline
\end{tabular}

\section{RESULTS AND}

\section{DISCUSSION}

The type of polymers (Eudragit NE 40D, Kollicoat SR 30D or Surelease E7) used as binder in granulation, the ratio of granulation polymers and the ratio of HPMC were formulation factors, whose influence on metoprolol release profile and type of kinetics was investigated using a full factorial experimental design. The in vitro release profiles of metoprolol tartrate from all the formulations prepared according to the design of experiment matrix are presented in Fig. 1. The all the pharmaceutical properties of the extended-release tablets (weight uniformity, tablets friability, tablets hardness) of the prepared formulations were within European Pharmacopeia limits.

Data fitting, coefficients calculation, statistical parameters evaluation and validation of the experimental design were performed using Modde software [19]. The Partial Least Squares [PLS] regression method was used for coefficients calculation and experimental data fitting. The values of R2 and Q2 were used to analyse the validity of the experimental design $[9,20]$.

Fig. 2 illustrates the overall fitting of the experimental data, as well as the statistical parameters R2 și Q2, model validity and reproducibility. The values of $R 2$ were greater than 0.7 for responses $Y 1-Y 8$, demonstrating a good fitting, and not a satisfactory fitting for the response Y9 (n Peppas).

Figure 3 presents the influence of the formulation variables on responses as coefficient plot and figure 4 presents the influence of the formulation variables on responses as contour plot surface.

The kinetic release of metoprolol from the prepared extended-release tablets was evaluated by using six mathematical models (Table 4). Table 5 shows the results obtained following fitting the data with different kinetic equations for all the prepared extended-release tablets (N1-N26). 

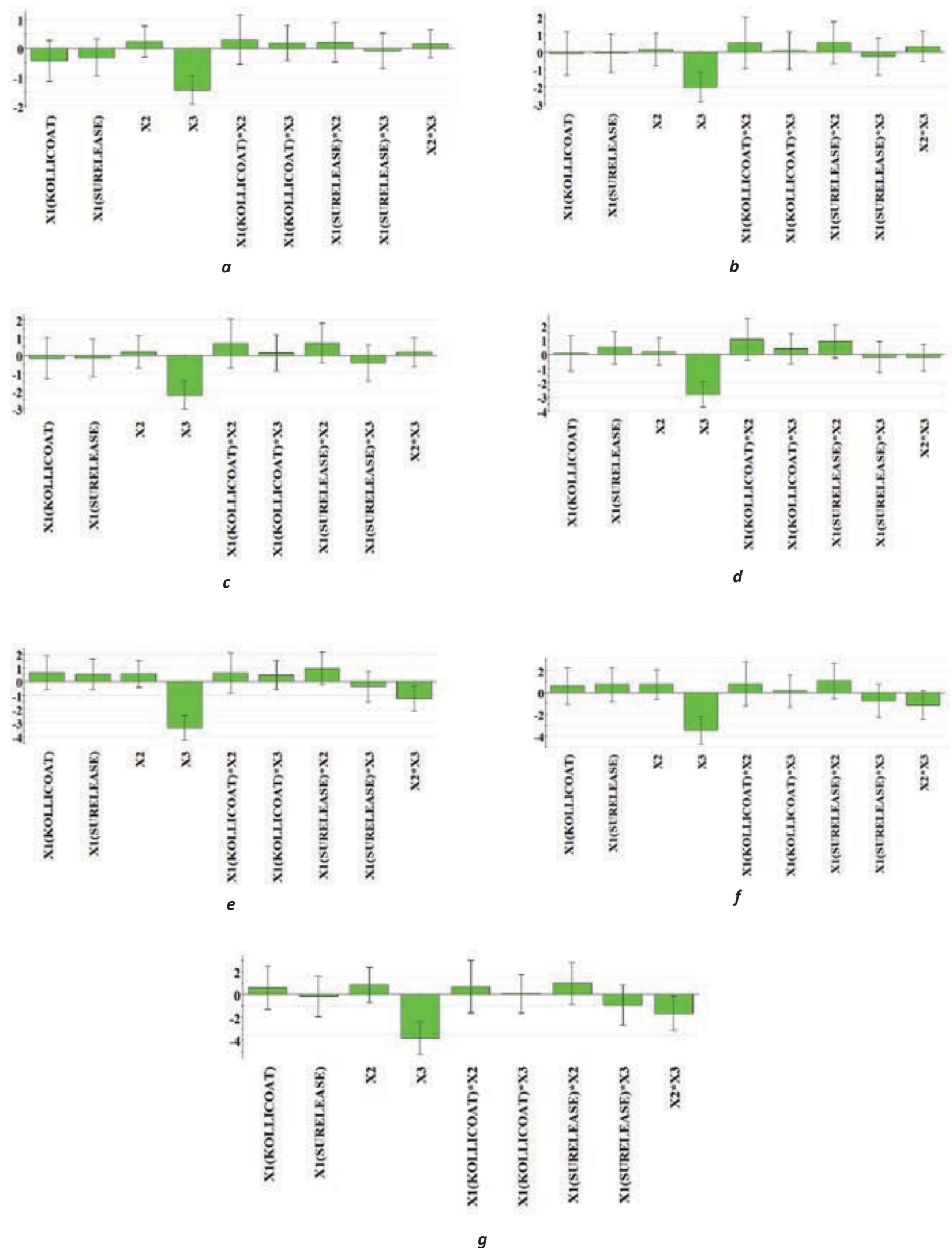

FIGURE 3. Coefficients' plots presentation illustrating the influence of formulation factors

$a-\%$ of metoprolol released after 1 hour (Y1), b-\% of metoprolol released after 2 hours (Y2), c - \% of metoprolol released after 4 hours (Y3), $d-\%$ of metoprolol released after 4 hours (Y4), $e-\%$ of metoprolol released after 6 hours (Y5), $f-\%$ of metoprolol released after 8 hours (Y6), g-\% of metoprolol released after 12 hours (Y7). X1 - type of granulation polymer, X2 - ratio of granulation polymer, X3 - HPMC ratio 
A
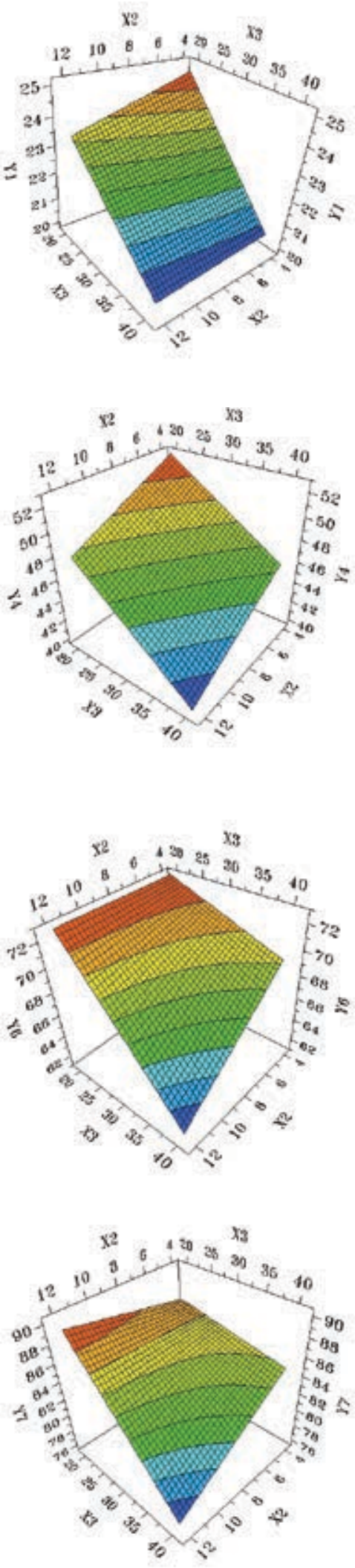

B
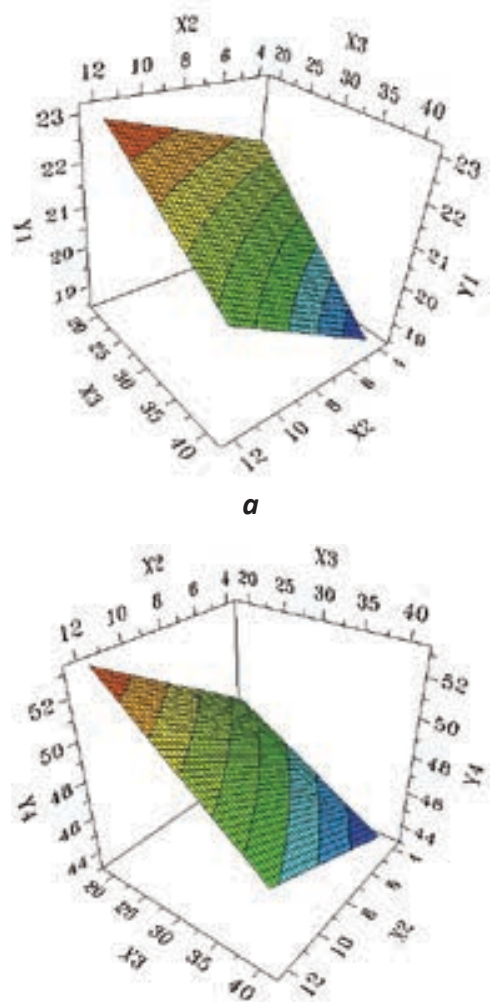

d
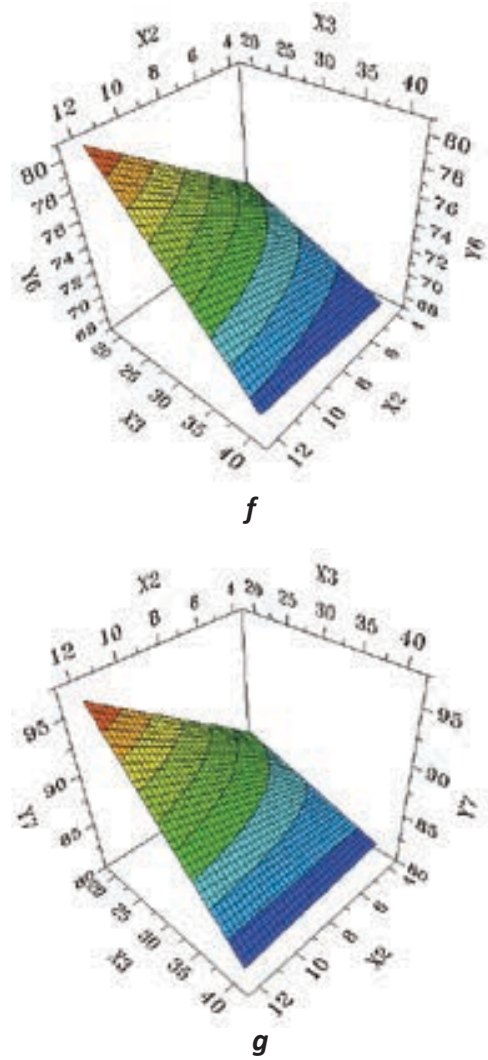

C
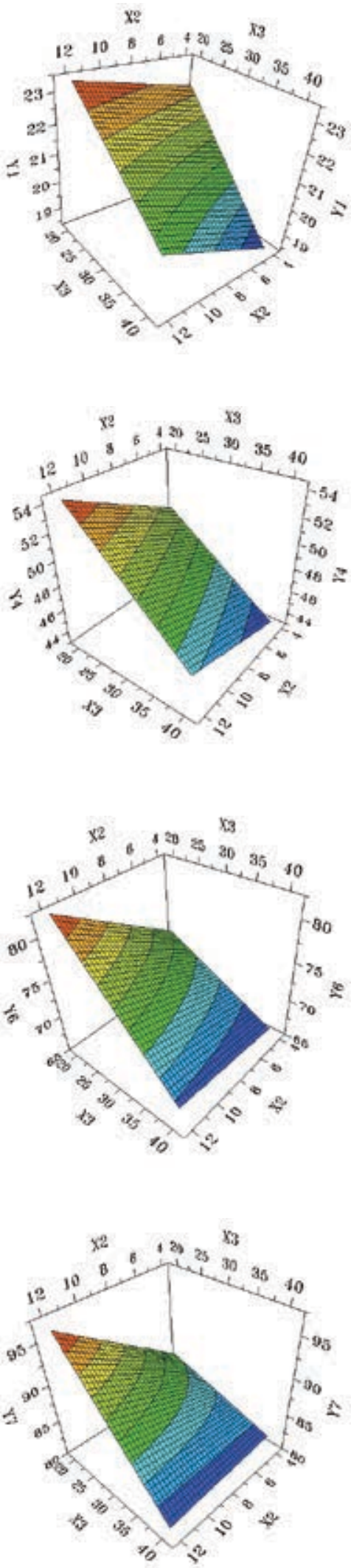

FIGURE 4. Contour plots illustrating the influence of formulation factors on metoprolol release 
TABLE 5. Results from fitting the dissolution profile with different kinetic equations

\begin{tabular}{|c|c|c|c|c|c|c|c|c|c|c|}
\hline \multicolumn{4}{|c|}{ Higuchi } & \multicolumn{4}{|c|}{ Korsmeyer - Peppas } & \multicolumn{3}{|c|}{ Zero order } \\
\hline & k & $r^{2}$ & AIC & $\mathbf{K}$ & n & $r^{2}$ & AIC & k & $r^{2}$ & AIC \\
\hline Exp 1 & 25.10 & 0.998 & 14.77 & 25.73 & 0.486 & 0.998 & 15.50 & 8.760 & 0.613 & 46.49 \\
\hline Exp 2 & 25.61 & 0.998 & 15.83 & 24.54 & 0.522 & 0.999 & 14.01 & 8.995 & 0.728 & 45.69 \\
\hline Exp 3 & 23.23 & 0.994 & 22.42 & 20.61 & 0.563 & 0.999 & 3.961 & 8.217 & 0.813 & 43.22 \\
\hline Exp 4 & 26.42 & 0.999 & 5.250 & 26.15 & 0.505 & 0.999 & 6.14 & 9.257 & 0.681 & 46.46 \\
\hline Exp 5 & 23.88 & 0.997 & 17.71 & 22.29 & 0.536 & 0.999 & 11.33 & 8.412 & 0.762 & 44.36 \\
\hline Exp 6 & 22.89 & 0.992 & 24.21 & 20.03 & 0.570 & 0.999 & 14.27 & 8.103 & 0.823 & 42.88 \\
\hline Exp 7 & 24.75 & 0.994 & 23.00 & 22.25 & 0.556 & 0.998 & 15.46 & 8.743 & 0.799 & 44.25 \\
\hline Exp 8 & 22.57 & 0.992 & 23.95 & 19.78 & 0.569 & 0.998 & 14.60 & 7.991 & 0.821 & 42.70 \\
\hline Exp 9 & 22.92 & 0.998 & 14.31 & 22.99 & 0.498 & 0.998 & 16.29 & 8.015 & 0.655 & 45.11 \\
\hline $\operatorname{Exp} 10$ & 23.51 & 0.997 & 17.65 & 22.12 & 0.532 & 0.998 & 14.41 & 8.277 & 0.752 & 44.29 \\
\hline Exp 11 & 25.07 & 0.997 & 18.65 & 25.29 & 0.495 & 0.997 & 20.58 & 8.765 & 0.644 & 46.28 \\
\hline Exp 12 & 23.42 & 0.987 & 28.29 & 19.42 & 0.598 & 0.998 & 16.78 & 8.325 & 0.861 & 42.37 \\
\hline Exp 13 & 23.09 & 0.989 & 31.08 & 19.56 & 0.587 & 0.998 & 16.73 & 8.196 & 0.847 & 42.56 \\
\hline Exp 14 & 25.63 & 0.992 & 25.77 & 22.41 & 0.571 & 0.998 & 16.16 & 9.071 & 0.823 & 44.31 \\
\hline Exp 15 & 25.71 & 0.991 & 27.20 & 21.96 & 0.583 & 0.999 & 13.67 & 9.121 & 0.842 & 43.92 \\
\hline Exp 16 & 23.04 & 0.997 & 17.86 & 22.55 & 0.511 & 0.997 & 19.42 & 8.074 & 0.695 & 44.85 \\
\hline Exp 17 & 23.96 & 0.998 & 15.24 & 22.84 & 0.525 & 0.999 & 12.17 & 8.422 & 0.735 & 44.81 \\
\hline Exp 18 & 23.80 & 0.996 & 20.52 & 22.04 & 0.540 & 0.998 & 17.08 & 8.381 & 0.768 & 44.37 \\
\hline Exp 19 & 21.78 & 0.993 & 22.84 & 19.56 & 0.556 & 0.997 & 18.44 & 7.688 & 0.798 & 42.89 \\
\hline Exp 20 & 26.94 & 0.995 & 23.55 & 24.87 & 0.542 & 0.997 & 21.36 & 9.491 & 0.770 & 45.87 \\
\hline Exp 21 & 25.48 & 0.995 & 22.63 & 23.65 & 0.539 & 0.997 & 20.96 & 8.972 & 0.764 & 45.29 \\
\hline Exp 22 & 22.70 & 0.994 & 22.09 & 20.86 & 0.545 & 0.997 & 19.83 & 8.000 & 0.775 & 43.75 \\
\hline Exp 23 & 27.56 & 0.996 & 22.17 & 26.50 & 0.521 & 0.996 & 23.10 & 9.672 & 0.720 & 46.80 \\
\hline Exp 24 & 24.67 & 0.993 & 23.97 & 22.08 & 0.559 & 0.998 & 17.82 & 8.716 & 0.803 & 44.25 \\
\hline Exp 25 & 22.18 & 0.997 & 15.98 & 21.05 & 0.527 & 0.999 & 13.59 & 7.798 & 0.740 & 43.8 \\
\hline Exp 26 & 25.72 & 0.989 & 27.58 & 23.07 & 0.557 & 0.994 & 26.25 & 9.081 & 0.797 & 44.98 \\
\hline & Hixo & d Crowell & & & Baker at & Lonsdal & & & irst orde & \\
\hline & k & $r^{2}$ & AIC & K & & & AIC & k & $r^{2}$ & AIC \\
\hline Exp 1 & 0.933 & 0.949 & 37.04 & 0.0156 & & & 27.27 & 0.178 & 0.972 & 31.86 \\
\hline Exp 2 & 0.961 & 0.950 & 34.70 & 0.0162 & & & 32.01 & 0.182 & 0.984 & 29.43 \\
\hline Exp 3 & 0.042 & 0.964 & 33.68 & 0.0126 & & & 32.90 & 0.150 & 0.984 & 28.95 \\
\hline Exp 4 & 0.053 & 0.952 & 35.94 & 0.0176 & & & 31.35 & 0.194 & 0.977 & 31.47 \\
\hline Exp 5 & 0.044 & 0.953 & 35.14 & 0.0136 & & & 31.52 & 0.159 & 0.978 & 30.65 \\
\hline Exp 6 & 0.041 & 0.967 & 33.21 & 0.0122 & & & 33.21 & 0.146 & 0.985 & 28.29 \\
\hline Exp 7 & 0.047 & 0.971 & 33.13 & 0.0148 & & & 34.01 & 0.169 & 0.986 & 28.54 \\
\hline Exp 8 & 0.040 & 0.964 & 33.47 & 0.0118 & & & 32.89 & 0.142 & 0.983 & 28.92 \\
\hline Exp 9 & 0.042 & 0.920 & 37.24 & 0.0124 & & & 32.89 & 0.149 & 0.963 & 32.64 \\
\hline Exp 10 & 0.043 & 0.946 & 35.76 & 0.0131 & & & 30.90 & 0.154 & 0.973 & 31.57 \\
\hline Exp 11 & 0.049 & 0.939 & 36.61 & 0.0155 & & & 29.01 & 0.177 & 0.974 & 31.53 \\
\hline Exp 12 & 0.043 & 0.983 & 29.93 & 0.0128 & & & 35.55 & 0.152 & 0.994 & 23.23 \\
\hline Exp 13 & 0.042 & 0.979 & 26.91 & 0.0124 & & & 34.57 & 0.148 & 0.993 & 24.34 \\
\hline Exp 14 & 0.050 & 0.983 & 30.69 & 0.0161 & & & 35.77 & 0.180 & 0.992 & 25.79 \\
\hline Exp 15 & 0.050 & 0.985 & 29.95 & 0.0161 & & & 36.60 & 0.180 & 0.992 & 26.33 \\
\hline Exp 16 & 0.042 & 0.934 & 36.43 & 0.0125 & & & 28.26 & 0.150 & 0.971 & 31.52 \\
\hline Exp 17 & 0.045 & 0.951 & 35.38 & 0.0138 & & & 30.40 & 0.161 & 0.979 & 30.21 \\
\hline Exp 18 & 0.044 & 0.962 & 34.15 & 0.0135 & & & 31.74 & 0.158 & 0.986 & 28.11 \\
\hline Exp 19 & 0.038 & 0.957 & 34.01 & 0.0108 & & & 31.16 & 0.135 & 0.984 & 28.25 \\
\hline $\operatorname{Exp} 20$ & 0.055 & 0.982 & 31.05 & 0.0184 & & & 35.17 & 0.200 & 0.993 & 25.40 \\
\hline Exp 21 & 0.050 & 0.974 & 32.74 & 0.0160 & & & 33.52 & 0.180 & 0.991 & 25.95 \\
\hline Exp 22 & 0.041 & 0.957 & 34.36 & 0.0120 & & & 31.19 & 0.146 & 0.984 & 28.51 \\
\hline Exp 23 & 0.058 & 0.979 & 32.02 & 0.0196 & & & 34.22 & 0.211 & 0.992 & 26.02 \\
\hline Exp 24 & 0.047 & 0.975 & 32.32 & 0.0147 & & & 34.09 & 0.168 & 0.991 & 26.18 \\
\hline $\operatorname{Exp} 25$ & 0.039 & 0.939 & 35.81 & 0.0114 & & & 28.64 & 0.140 & 0.973 & 31.01 \\
\hline $\operatorname{Exp} 26$ & 0.051 & 0.978 & 32.20 & 0.0163 & & & 35.63 & 0.182 & 0.990 & 27.31 \\
\hline
\end{tabular}




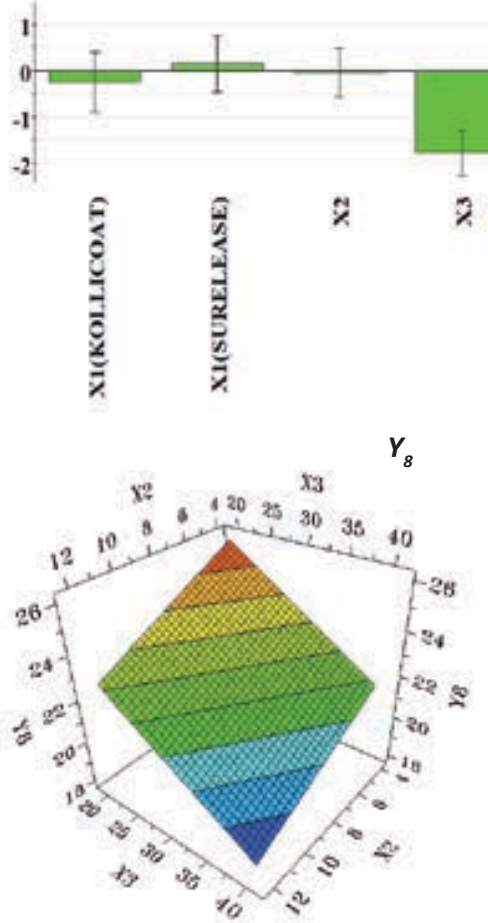

A

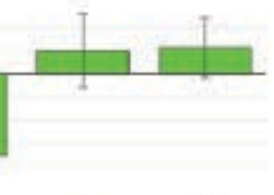

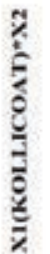
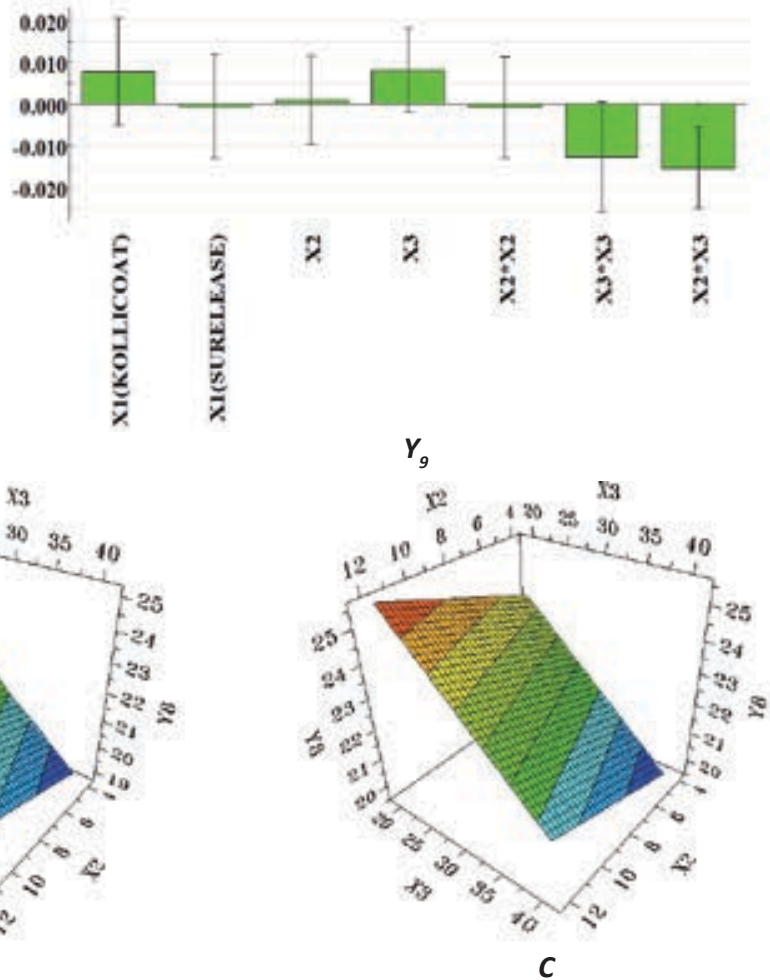

FIGURE 5. Regression of coefficients' plots and contour surface plots showing the influence of the formulation factors on kinetic release parameters

X1 - Granulation polymer type, X2 - ratio of granulation polymer, X3 - HPMC ratio; Y8 - k Peppas, Y9-n Peppas

A-Eudragit; $B$ - Kollicoat; $C$-Surelease

The coefficients of the equation used to fit the experimental data with the chosen model for kinetic release evaluation are present in fig. 5 scaled and centred plots (in Fig. 5, Y8 and Y9,) and as response surface plot (in Fig. 5. A, B, C).

Fig. 3 and Fig. 4 illustrate, in the form of coefficient plots and contour plot surfaces, respectively, the effect which the formulation factors and their interaction had on the release of metoprolol from the extended-release tablets. The coefficients analysis revealed that HPMC ratio (X3) is the main factor that affected metoprolol's release, namely an increase in the amount of Methocel K100 M reduced the drug release rate at all dissolution time points (Fig 3. a-g). As respects the influence of the polymer type (X1) used for the granulation process, only Eudragit reduced the dissolution rate of drug released at all time points (Fig 4A), and the influence of Kollicoat and Surelease are dependent on the HPMC ratio and the dissolution time point (Fig. 4B - Kollicoat; Fig. 4CSurelease). The influence of the binder polymer ratio on metoprolol released was different, depending on the granulation polymer type (X1). A greater amount of Eudragit decreased the percentage of metoprolol released at all dissolution time points (Fig. 4A), while increasing the amount of Kollicoat and Surelease increased the percentage of metoprolol released in the first 6 hours (Fig. 4B-a,d; Fig 4C-a,d) and had no effect after 6 hours if a high amount of HPMC was used (Fig. 4B-e,g; Fig 4C-e,g). The fact that the drug release increased instead of decreasing at higher Kollicoat and Surelease concentrations may be explained by the following: when increasing granulation polymer amount (Kollicoat or Surelease), the polymer layer becomes thicker and its plasticity is reduced. As a result, the polymer's flexibility decreases, its brittleness increases and determines its rupture at the time of compression.

\section{DISCUSSION}

According to literature data, ethylcellulose appears to be a more brittle polymer than acrylic polymers, therefore the latter are preferred for coating of multiparticulate systems before their compression, due to their increased flexibility [21]. Similar results were obtained by other researchers, regarding the drug release kinetics profile by different polymers. Nellore et al. examined the metoprolol kinetics release from hydrophilic matrix tablets prepared with different percent of HPMC, ranging from $10 \%$ to $40 \%$. 
They observed that an increase of the amount of HPMC from $10 \%$ to 40 in hydrophilic matrix tablets prepared via direct compression, conduct to a significant reduction of the metoprolol tartrate release rate. However, for hydrophilic matrix tablets prepared trough fluid bed granulation, the influence was less clear [20]. Muschert et al. obtained different results from the ones obtained in the present study, namely they described a reduction of the diltiazem release rate from coated pellets with ethylcellulose [22].

The kinetic release of metoprolol tartrate from the extended-release hydrophilic matrix tablets was evaluated using six mathematical models (presented in Table IV). Table V shows the results and the statistical parameters obtained at kinetics release characterization. All formulations (N1 - N26) fitted best with the Peppas model from the kinetic release profile point of view. Following this finding, $k$ and $n$ parameters of Peppas equation were introduced as responses ( $Y 8-k$ and $Y 9-n$ ) of the DoE in order to evaluate the effect of the studied formulation factors on the metoprolol kinetic release. Fig. 5 revealed that the HPMC ratio (X3) was the only factor that influenced the parameter $k$ Peppas, namely the value of $k$ Peppas decreased when the HPMC ratio increased. The influence of the ratio of the polymer and the type of polymer used for the granulation process (Eudragit, Kollicoat, and Surelease) were insignificant. The analysis of coefficients did not identify any interactions between the studied formulation factors. The percentage of the drug released was different depending on the granulation polymer type (Fig. 5): increasing the amount of Eudragit reduced k Peppas, while increasing the amount of Kollicoat and Surelease increased k Peppas. The formulation factors had a similar influence on the parameter $k$ Peppas as on the in vitro release of metoprolol tartrate. The results obtained by other researchers were similar with the ones obtained in this study, showing that an enhancement of polymer concentration (Kollidon SR) decreased k Peppas release constant [29]. The studied formulation factors have not influenced on the $n$ Peppas parameter, as no correlation between the formulation factors and $n$ Peppas parameter was identified. All the values of $n$ are close to 0.5 (with low variability between 0.48 and 0.58 ) that suggest a system with Higuchi kinetic release behaviour.

Based on scientific literature information, matrix tablets with HPMC or other hydrophilic polymers determine a complex drug kinetic release, which includes swelling, diffusion and erosion steps and is based on Korsmeyer-Peppas kinetic release mathematical model [23-29]. Generally, the drug release from hydrophilic matrix tablets takes place in three steps: (1) the infiltration of the dissolution medium into the matrix and its hydration; (2) the swelling and the erosion of the matrix; (3) the transfer of the dissolved drug through the hydrated and swollen matrix, or the transfer of matrix fragments into the dissolution medium $[4,29]$. However, the results of this study showed that drug release occurs after a Higuchi model (n Peppas model's mechanism for a $\mathrm{n}$ value close to 0.5 is similar to Higuchi's model) [30]. Therefore, the extended-release tablets obtained with the two types of polymers (high viscosity HPMC and Eudragit / Kollicoat / Surelease) appear to determine a release which happens after a Higuchi model $[25,27,30]$. Shoaib et al. obtained similar results, namely observed a release kinetics which happened after a Higuchi model for ibuprofen HPMC matrix tablets [24]. Finally, according to $n$ Peppas, metoprolol exhibited a kinetic release from the prepared tablets which consisted in the diffusion and erosion of the matrix $[25,30]$.

\section{CONCLUSIONS}

In this study, hydrophilic HPMC matrix extendedrelease tablets were developed using design of experiments. Three formulation factors were chosen to be studied - type of granulation polymer, ratio of granulation polymer and HPMC ratio, in order to evaluate their influence on the metoprolol kinetic release. The conclusions that could be drawn from this study are the following: HMPC ratio (X3) was the main factor that affected drug release during the 12 hours dissolution test, as the results showed a reduction of the released drug percentage at all dissolution points following the increase of HPMC ratio; regarding the polymer type and ratio, Eudragit was the only polymer used as binder in fluid bed granulation that determined the decrease of the amount of metoprolol released at all dissolution points, while increasing the ratio of Kollicoat and Surelease determined an increase of the percent of metoprolol released in the first 4 hours, but did not influence the release after 6 hours for formulations with a high amount of HPMC; the kinetic release fitted best with Peppas model for all formulations, and the value of $n$ of Peppas model was close of 0.5, which proves that metoprolol release was determined 
by drug diffusion and matrix erosion, according to a Higuchi model.

In conclusion, it is possible to reduce the burst effect from hydrophilic matrix extend release dosage forms with highly soluble drug (as metoprolol) if the drug is granulated with a high amount of Eudragit NE 40D and the obtained granules are incorporated in the matrix by tableting.

\section{Acknowledgements}

Special thanks to COLORCON (from UK) for providing free Methocel and Surelease samples, to Degussa/ Evonik (from Germany) for providing free Eudragit samples and to BASF (from Germany) for providing free Kollicoat samples.

This work was also supported by the "Iuliu Hatieganu" University of Medicine and Pharmacy Cluj-Napoca, project number 27020/4/11.2011.

Conflict of interest: none declared Financial support: none declared

\section{REFERENCES}

1. Mujtaba A, Kohli K. In vitro/in vivo evaluation of HPMC/alginate based extended-release matrix tablets of cefpodoxime proxetil. Int J Biol Macromol. 2016;89:434-441.

2. Ofori-Kwakye K, Mfoafo KA, Kipo SL. Development and evaluation of natural gum-based extended-release matrix tablets of two model drugs of different water solubilities by direct compression. Saudi Pharm J. 2016;24:82-91.

3. Li L, Wang L, Li J etc. Insights into the mechanisms of chitosananionic polymers-based matrix tablets for extended drug release. Int J Pharm. 2014;476:253-265.

4. Grassi M, Grassi G. Mathematical Modelling and Controlled Drug Delivery: Matrix Systems. Curr Drug Deliv. 2005;2(1):97-6.

5. Siddique S, Khanam J, Bigoniya P. Development of Sustained Release Capsules Containing "Coated Matrix Granules of Metoprolol Tartrate". AAPS PharmSciTech. 2010;11:1306-1314.

6. Shao ZJ, Moralesi L, Diaz S etc. Drug Release From Kollicoat SR 30D-Coated Nonpareil Beads: Evaluation of Coating Level, Plasticizer Type, and Curing Condition. AAPS PharmSciTech. 2002;3(2):E15.

7. Siepmann F, Hoffmann A, Leclercq B etc. How to adjust desired drugs release patterns from ethylcellulose-coated dosage forms. J Control Release. 2007;119(2):182-189.

8. Muschert S, Siepmann F, Leclercq B etc. Prediction of drug release from ethylcellulose coated pellets. J Control Release.2009;135(1):71-79.

9. Bodea M, Tomuţa I, Leucuţa SE. Identification of critical formulation variables for obtaining metoprolol tartrate mini-tablets. Farmacia. 2010;58:719-727.

10. Vignaduzzo SE, Castellano PM, Kaufman TS. Development and Validation of a Dissolution Test for Meloxicam and Pridinol Mesylate from Combined Tablet Formulation. Indian J Pharm Sci. 2010;72(2):197-203.

11. Tomuta I, Leucuta SE. The influence of formulation factors on the kinetic release of metoprolol tartrate from prolong release coated minitablets. Drug Dev Ind Pharm. 2007;33(10):1071-1087.

12. Barka N, Abdennouri M, Boussaoud A etc. Full factorial experimental design applied to oxalic acid photocatalytic degradation in TiO2 aqueous suspension. Arab J Chem. 2014;7:752757.

13. Suchana A, Passeport E. Optimization of a solid-phase microextraction technique for chloro- and nitro- substituted aromatic compounds using design of experiments. J Chromatogr $A$. 2020;1621:461083.

14. Kurmi M, Jayaraman K, Natarajan S etc. Rapid and efficient chiral method development for lamivudine and tenofovir disoproxil fumarate fixed dose combination using ultra-high performance supercritical fluid chromatography: A design of experiment approach. J Chromatogr A. 2020;1625:461257.
15. Eriksson L, Johansson E, Kettaneh-Wold N etc. Design of Experiments: Principles and Applications. Umetrics Academy, Umea 2000.

16. Thakkar VT, Shah PA, Soni TG etc. Goodness-of-Fit ModelDependent Approach for Release Kinetics of Levofloxacin Hemihydrates Floating Tablet. Dissolut Technol. 2009;16:35-43.

17. Sood A, Panchagnula R. Role of dissolution studies in controlled release drug delivery systems. S.T.P. Pharm Sci. 1999;9:157-168.

18. Prasacu I, Mircioiu C, Sandulovici R etc.Release of metoprolol from solid dosage forms. Choice and validation of theoretical model. Farmacia. 2009;57(1):89-98.

19. MODDE 9, Software for Design of Experiments and Optimization: User's Guide and Tutorial, Umetrics Academy Inc, Umea 2010.

20. Nellore RJ, Rekhi GS, Hussain AS etc. Development of metoprolol tartrate extended-release matrix tablet formulations for regulatory policy consideration. J Control Release. 1998;50(1-3):247-256.

21. Zoubari G, Ali R, Dashevskiy A. Water-insoluble polymers as binders for pellet drug layering: Effect on drug release and performance upon compression. Int J Pharm. 2019;569:118520.

22. Muschert S, Siepmann F, Leclercq B etc. Prediction of drug release from ethylcellulose coated pellets. J Control Release. 2009;135(1):71-79.

23. Achim M, Tefas LR, lovanov R, Vonica-Gligor AL, Barbu-Tudoran L, Tomuță I, Preparation and in vitro evaluation of felodipine-loaded poli ( $\varepsilon$ caprolactone) microspheres: quality by design approach. Farmacia, 67 (4):670-683.

24. Shoaib MH, Tazeen J, Merchant HA etc. Evaluation of drug release kinetics from ibuprofen matrix tablets using HPMC. Pak J Pharm. Sci. 2006;19(2):119-124.

25. Siepmann J, Siepmann F. Mathematical modeling of drug delivery. Int J Pharm. 2008;364(2):328-343.

26. Vrbanac $H$, Krese $A$. The influence of different mechanical stress on the release properties of HPMC matrix tablets in sucrose- $\mathrm{NaCl}$ media. J Drug Deliv Sci Technol. 2019;54:101246.

27. Gâvan A, Porfire A, Marina C, Tomuță I, Formulation and pharmaceutical development of quetiapine fumarate sustained release matrix tablets using a QbD approach., Acta Pharmaceutica 2017; 67:53-70.

28. Fukui $\mathrm{S}$, Yano $\mathrm{H}$, Yada S etc. Design and evaluation of an extendedrelease matrix tablet formulation; the combination of hypromellose acetate succinate and hydroxypropylcellulose. Asian J Pharm Sci. 2017;12:149-156.

29. Cheng Y, Qin H, Acevedo NC etc. 3D printing of extended-release tablets of theophylline using hydroxypropyl methylcellulose (HPMC) hydrogels. Int J Pharm. 2020;591: 119983.

30. Gohel MC, Panchal MK, Jogani VV. Novel Mathematical Method for Quantitative Expression of Deviation from Higuchi Model. AAPS PharmSciTech. 2000;1(4):E31. 